\title{
Un modelo cualitativo de la comunidad biológica del Norte del Golfo de California, México: posibles efectos de la explotación pesquera en el ecosistema
}

\author{
A qualitative model for the biological community of the \\ Northern Gulf of California, Mexico: potential fisheries effects \\ on the ecosystem
}

\author{
Alejandro Espinoza-Tenorio*1, Gabriela Montaño-Moctezuma ${ }^{2}$ y Ileana Espejel ${ }^{3}$
}

\begin{abstract}
RESUMEN
El manejo basado en ecosistemas (MBE) es uno de los principales paradigmas bajo los cuales se están rediseñando las pesquerías mundiales. Sin embargo, la implementación exitosa del MBE se ha visto limitada debido a la complejidad intrínseca de los sistemas ecológicos y a la necesidad de tener suficiente información para construir y analizar modelos complejos. Para implementar exitosamente un MBE en pesquerías, países como México necesitan diseñar y promover herramientas de manejo acordes a su contexto social y natural, y así aprovechar de las capacidades de su sistema pesquero. En este trabajo se utilizó un modelo cualitativo (Loop Analysis) para visualizar las implicaciones ecosistémicas de la explotación pesquera sobre algunos de los miembros de una comunidad marina. Con base en seis grupos de interés pesquero y un grupo de interés para la conservación, construimos un modelo sobre la comunidad biológica sujeta a pesca del Norte del Golfo de California (NGC). El análisis de las predicciones del modelo permitió conocer los efectos directos e indirectos de la explotación de diferentes grupos pesqueros, así como identificar las pesquerías que pueden presentar conflicto al operar de manera simultánea. La naturaleza cualitativa del modelo permitió incorporar como fuente de información el conocimiento ecológico local (CEL), propiciando así el futuro involucramiento de los actores en el diseño de estrategias de manejo. Las ideas generadas pueden ser útiles para establecer objetivos tanto de utilización de los recursos como de conservación, y proporcionan una guía para elaborar propuestas de manejo basadas en el conocimiento del ecosistema.
\end{abstract}

Palabras claves: Manejo con base en ecosistemas, conocimiento ecológico local, análisis cualitativo, pesca artesanal, Golfo de California.

\begin{abstract}
Ecosystem-based management (EBM) is one of the main paradigms under which the world's fisheries have been redesigned. However, successful implementation of EBM has been limited due to the inherent complexity of ecological systems as well as the need to have enough information to build and analyze complex models. To successfully implement EBM in fisheries, countries such as Mexico need to design and promote management tools consistent with their social and natural context in a way that they can take advantage of the capabilities of their fishery

\footnotetext{
Facultad de Ciencias Marinas e Instituto de Investigaciones Oceanológicas, UABC. Adscripción actual: El Colegio de la Frontera Sur (ECOSUR)-Unidad Villahermosa, Carr. Reforma 15.5 km, 86280, Villahermosa, México, aespinoza@ecosur.mx*.

2 Instituto de Investigaciones Oceanológicas (IIO), Universidad Autónoma de Baja California (UABC), Carretera TijuanaEnsenada 103 km, 22800, Ensenada, México, gmontano@uabc.edu.mx.

3 Facultad de Ciencias, Universidad Autónoma de Baja California (UABC), Carretera Tijuana-Ensenada 103 km, 22800, Ensenada, México, Ileana.espejel@uabc.edu.mx
}

Recibido: 15 de noviembre de 2014

Corregido: 12 de mayo de 2015

Aceptado: 19 de junio de 2015

DOI: http://dx.doi.org/10.15359/revmar.7.4

Rev. Mar. Cost. ISSN 1659-455X. Vol. 7: 53-72, Diciembre 2015. 
systems. In this paper we used a qualitative model (Loop Analysis) to examine the ecosystem implications of fishery on some members of a marine community. A model for the biological community of the Northern Gulf of California (NGC) was constructed, based on six fishery groups and a conservation group. Model predictions allowed assessing both direct and indirect effects from harvesting different fishery groups, as well as identifying those fisheries that might present a conflict when operating simultaneously. The qualitative nature of the model allowed incorporating local ecological knowledge (LEK) as a source of information, fostering the future involvement of stakeholders in the design of management strategies. The proposed ideas may be useful to generate goals regarding resource exploitation and conservation and also provide a guide to generate management strategies based on the knowledge of the ecosystem.

Keywords: Ecosystem-based management, local ecological knowledge, qualitative analysis, artisanal fisheries, Gulf of California.

\section{INTRODUCCIÓN}

El grave y creciente deterioro de los recursos pesqueros marinos ha llevado a cuestionar la continuidad del modelo de aprovechamiento imperante que se basa en la creencia de que los ambientes marinos son inalterables y sus recursos ilimitados y controlables (Finley, 2009). En la búsqueda de un nuevo sistema de manejo que garantice el uso de los recursos sin comprometer su conservación a largo plazo, durante las últimas tres décadas la comunidad científica internacional ha impulsado el manejo basado en ecosistemas (MBE) como el marco conceptual más prometedor para rediseñar las pesquerías (Pikitch et al. 2004). Tal rediseño del manejo pesquero ha significado adoptar paradigmas de aprovechamiento integral que ayuden a un mejor entendimiento no solo del recurso aprovechado, sino de todo el ecosistema- incluyendo al hombre- en el que se encuentra inserto. De manera que se han diseñado una serie de herramientas para lidiar con los nuevos retos metodológicos que implica el manejo de sistemas en su conjunto. Los modelos ecosistémicos son una de estas herramientas, y han recibido especial atención por científicos y manejadores pesqueros porque permiten evaluar las potenciales respuestas de los ecosistemas ante distintos impactos ambientales o estrategias de manejo (Plagányi, 2007).

Aplicar el MBE ha sido más difícil de lo que se pensaba (Caddy \& Seijo, 2005; Corkeron, 2006; Levin \& Lubchenco, 2008). Especialmente en países en desarrollo como México, donde a la complejidad intrínseca del MBE se suman la problemática de información cuantitativa escasa e inaccesible, recursos humanos insuficientes y poco calificados, pescadores y científicos no involucrados en la toma de decisiones (Zárate et al. 1999; Ortiz-Lozano et al. 2005). No obstante, a diferencia del sistema de manejo poblacional, el MBE promueve la integración de todos los actores sociales involucrados en la toma de decisiones, lo cual permite mejorar las estructuras de gobernanza sobre los recursos marinos (Ruckelshaus et al. 2008) y así diseñar estrategias más acordes a las 
circunstancias locales (CBD, 2003). Para el escenario pesquero mexicano, una mayor participación de los distintos actores sociales significaría un mejor aprovechamiento tanto de sus capacidades técnicas, administrativas y científicas, como de su conocimiento empírico sobre los recursos pesqueros y los ecosistemas donde se encuentran (Hernández \& Kempton, 2003).

El Norte del Golfo de California (NGC) forma parte de una de las regiones marinas con mayor biodiversidad del planeta (IUCN, 2005) y es uno de los ecosistemas marinos más estudiados en México; incluso es uno de los pocos con varios trabajos cuantitativos de modelación ecosistémica (ArreguínSánchez et al. 2002; Morales-Zárate et al. 2004; Lercari, 2006). Para regular los impactos de las dos flotas pesqueras (artesanal e industrial) que compiten en el NGC se han implementado medidas de manejo como las vedas, talla mínima, control de permisionarios y el establecimiento de la Reserva de la Biosfera Alto Golfo de California y Delta del Río Colorado (RBAGCyDRC). Sin embargo, dichos esfuerzos han sido insuficientes y existe amplia evidencia de una marcada disminución en la abundancia de recursos pesqueros (Cinti et al. 2014). Aunque las causas son múltiples (e.g., cambios en el aporte de agua dulce del Río Colorado, la piratería y la consideración aislada de las actividades humanas), se ha sugerido que durante la planeación de las políticas pesqueras no se ha dado a conocer adecuadamente a los pescadores de la importancia de su participación (Bobadilla et al.2011). A esto se suman las limitaciones técnicas para emplear a los modelos ecosistémicos cuantitativos en la toma de decisiones (e.g., necesidad de grandes series de datos cuantitativos, poca accesibilidad a un amplio grupo de usuarios, largo tiempo de construcción; Bondavalli et al. 2009; Espinoza-Tenorio et al. 2012).

En el escenario antes descrito, el presente estudio tuvo como objetivo utilizar un modelo trófico cualitativo (Loop Analysis por su nombre en inglés) para conocer los efectos directos e indirectos de la explotación pesquera en el Norte del Golfo de California (NGC).

\section{MATERIAL Y MÉTODOS}

\section{Recopilación del conocimiento ecológico local}

El conocimiento ecológico local (CEL) es aquella información que se tiene sobre un ecosistema específico y que puede estar constituida por una mezcla de datos de origen tanto científico como empírico (Berkes \& Folke, 2002). El CEL es el conocimiento proveniente de la observación de especies y otros fenómenos ambientales que, aunque puede tratar sobre procesos históricos a largo plazo y transgeneracionales, ha sido adquirido durante el periodo de vida de individuos (Gilchrist et al. 2005). Para recopilar el conocimiento pesquero y trófico sobre la comunidad biológica del NGC (Cuadros 1 y 2) se integró información cuantitativa y cualitativa. La primera fue tomada directamente de las bases de datos pesqueros oficiales y de literatura científica publicada y gris (reportes técnicos y tesis). 
Cuadro 1. Información pesquera que se utilizó para generar los modelos sobre la comunidad biológica del Norte del Golfo de California, México, de acuerdo con tres tipos de grupos pesqueros y de conservación: a) especies objetivo, b) captura incidental y c) especies protegidas. La producción total de los principales recursos representa la captura acumulada de seis años (1993-1998)

Table 1. Fishing information selected to create the models for the biological community of the Northern Gulf of California, Mexico according to three types of fishery and conservation groups: a) target species, b) by-catch, and c) protected species. Total production of the main resources represents the accumulated capture during six years (1993-1998)

\begin{tabular}{|c|c|c|}
\hline $\begin{array}{l}\text { Grupo } \\
\text { (producción }{ }^{(8)} \text { ) }\end{array}$ & a) Especies objetivo & $\begin{array}{l}\text { Fuentes de } \\
\text { información }\end{array}$ \\
\hline $\begin{array}{l}\text { Crustáceos } \\
(23000 \mathrm{t})\end{array}$ & $\begin{array}{c}5 \text { especies: Camarón (Litopenaeus stylirostris, L. californiensis) } \\
\text { y cangrejo (Callinectes belicosus, C. arcuatus, C. toxotes) }\end{array}$ & I, III, V, VII, IX \\
\hline $\begin{array}{l}\text { Escama } \\
(21700 \mathrm{t})\end{array}$ & $\begin{array}{l}5 \text { especies: Chano (Micropogonias megalops), corvina (Cynoscion } \\
\text { othonopterus), sierra (Scomberomorus concolor), baqueta } \\
\text { (Epinephelus acanthistius) y extranjero (Paralabrax auroguttatus) }\end{array}$ & I, III, V, VII, IX \\
\hline $\begin{array}{l}\text { Elasmobranquios } \\
(8900 \mathrm{t})\end{array}$ & $\begin{array}{l}5 \text { especies: Tiburones (Squatina californica, Rhizoprionodon } \\
\text { longurio, Mustelus lunulatus), guitarra (Rhinobatus productus) } \\
\text { y mantarraya (Dasyatis brevis) }\end{array}$ & I, III, V, VII, IX \\
\hline $\begin{array}{l}\text { Moluscos } \\
(1800 t)\end{array}$ & $\begin{array}{l}10 \text { especies: Almeja (Argopecten circularis, Tibela stultorum), ostión } \\
\text { (Crassostrea corteziensis), caracol (Hexaplex nigritus, Phyllonotus } \\
\text { erythrostoma, Astrea undosa, A. turbanica), pulpo (Octopus } \\
\text { bimaculatus, O. hubbsorum) y calamar (Dosidicus gigas) }\end{array}$ & I, III, V, VII, IX \\
\hline
\end{tabular}

\section{b) Captura incidental}

Carnívora

$(1448 \mathrm{t})$

Omnívora

$(174 \mathrm{t})$

40 especies: Lenguado (Paralichthys spp.), cochito (Pseudobalistes spp.), merluza (Merluccius productus), cabrilla (Epinephelus spp.), pez guitarra (Rhinobatos spp.), botete (Tetrodon III, IX annulatus), otras*

11 especies: Lisa (Mugil cephalus), mojarra (Eucinostomus spp.), callo (Spondylus calcifer, Pteria sterna, P. rugosa, Atrina tuberculos, Spondylus princeps), mejillón (Modiolus capax), madreperla (Pinctada mazatlanica) y pepino de mar (Isostichopus fuscus, Parastichopus parvimensis)

\section{c) Especies protegidas}

7 especies: Totoaba (Totoaba macdonaldi), tortugas marinas

Especies protegidas (NR) (Lepidochelis olivacea, Chelonia agassizi), vaquita marina 1, 2-7 / I, II, IV, V, (Phocoena sinus), delfines (Tursiups spp., Delphinus delphis) y VI, VII, VIII león marino (Zalophus californianus)

$\mathrm{NR}=$ No registrada. $*$ Captura incidental carnívora con reportes menores a $20 \mathrm{t}$ : boca dulce o berrugata (Menticirrhus nasus), pargo (Lutjanus spp.), jurel (Caranx hippos), cabaycucho (Diplectrum pacificum), pampano (Trachinotus spp.), pez puerco (Balistes polylepsis), pierna o conejo (Caulolatilus spp.), bacoco (Haemulon spp.), baya (Mycteroperca jordani), lengua (Lepophidium prorates), gallo (Nematistius pectoralis), mero (Serranus niveatus), barracuda mexicana (Sphyraena ensis y S. argentea), bichi (Oligoplites spp.), conejo (Caulolatilus affinis), mahi-mahi (Coryphaena hippurus), manta (Raja spp., Mobula spp., Gymnura marmorata y Myliobatis californica), pez gallo (Caranx otrynter), barbudo (Polydactylus approximans) y otros tiburones (Alopias spp., Carcharhinus leucas, Sphyrna spp., C. obscurus, Heterodontus spp., Negaprion brevirostris, S. mokarran, Isurus oxyrinchus, Galeocerdo cuvier, Carcharodon carcharia, Carcharhinus limbatus). (Fuentes de información. Cuantitativa: a) Literatura publicada: 1. Cisneros-Mata et al. (1995); 2. CITES (2011); 3. DOF (1990); 4. DOF (1994); 5. IUCN (2011); 6. Pedrín-Osuna et al. (2001); 7. DOF (2007) y b) Literatura gris: 8. SAGARPA (1998). Cualitativa: a) Informantes clave: I. Cudney, R. y II. Turk P. CEDO (comunicación personal, enero, 2004); III. García, J. M. antes con Conservación Internacional (comunicación personal, enero, 2004); IV. Campoy, F. J. RBAGCyDRC (comunicación personal, enero, 2004), b) Grupos especializados: V. Administradores, RBAGCyDRC; VI. Investigadores, CoBi, c) Reuniones especializadas: VII. Sesión de la mesa directiva de la RBAGCyDRC; VIII. Reunión del Comité Internacional para la Recuperación de la Vaquita Marina (CIRVA) y d) Entrevistas a pescadores: IX. Cudney \& Turk (1998). 
El conocimiento adquirido empíricamente por los usuarios (pescadores, investigadores, administradores de recursos naturales y conservacionistas) sobre los ecosistemas pesqueros fue recopilado mediante el uso de técnicas de las ciencias sociales que emplean la interacción entre el investigador y los informantes clave como un medio no intrusivo para obtener datos de un modo sistemático (Taylor \& Bogdan, 1990). Las tres técnicas utilizadas para recopilar el conocimiento cualitativo fueron: a) diez entrevistas semiestructuradas a investigadores pesqueros, activistas por la conservación y tomadores de decisiones sobre los recursos pesqueros (hábitos alimenticios, estado poblacional y principales impactos), b) cinco talleres a grupos de investigación pesquera, activistas por la conservación y administradores de la RBAGCyDRC, donde se presentaron y discutieron resultados preliminares del modelo, y c) participación en dos eventos especializados sobre la problemática del manejo de la pesca y la conservación de la vaquita marina (Phocoena sinus) en el NGC. El conocimiento de los pescadores fue incorporado a través de los resultados del estudio de Cudney \& Turk (1998) en el NGC, quienes realizaron 117 entrevistas estructuradas y 170 no estructuradas a pescadores, directivos de cooperativas, comerciantes y personal de la RBAGCyDRC.

\section{Análisis cualitativo (Loop Analysis)}

El método de modelación Loop Analysis (por su nombre en inglés; Puccia \& Levins, 1985) utiliza las interacciones entre variables para representar un sistema a través de un diagrama de signos. Las interacciones o relaciones entre variables se representan mediante cabezas de flecha $(\rightarrow)$ o cabezas de círculos $(-\bullet)$ y significan la relación positiva (+) o negativa (-) que existe entre variables, respectivamente. Desde un punto de vista biológico, una flecha significa el beneficio que recibe un depredador al consumir a su presa, y por el contrario, una cabeza de círculo describe el efecto negativo que recibe la presa al ser consumida. Los efectos de autorregulación -positivos (flechas) o negativos (círculos)- se indican como ligas que inician y terminan en la misma variable y representan a todos aquellos procesos o fenómenos (internos o externos) que no son incluidos específicamente en el sistema construido, pero regulan a las variables (e.g., denso-dependencia, aporte de larvas o reclutas de otro sistema, el sistema de nutrientes asociado a las algas).

Los sistemas o modelos tróficos se representan por un diagrama de signos (Fig. 1a). En este ejemplo de tres variables, los peces $(\mathrm{P})$ consumen erizos (E), que a su vez comen algas (A). Las interrelaciones entre variables se introducen como elementos $\left(a_{i j}\right)$ para construir una matriz de la comunidad $[\mathrm{A}]$. 


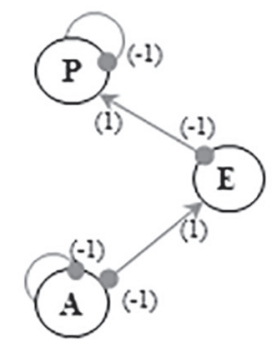

a)

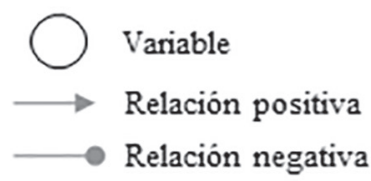

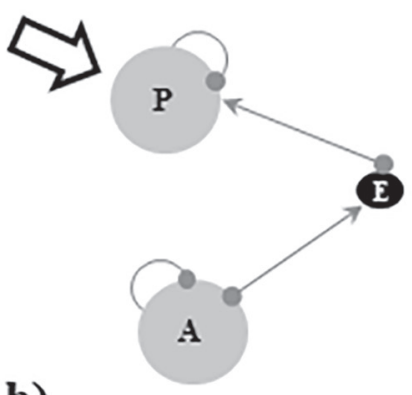

b)

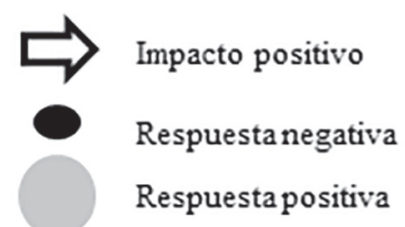

Fig. 1. a) Diagrama de signos de un sistema trófico sencillo de tres variables ( $\mathrm{P}=\mathrm{Peces}, \mathrm{E}=\mathrm{E}=\mathrm{zizos}$ y A=Algas). La autorregulación negativa en los peces significa que la población consume presas alternativas que no fueron incluidas en el modelo; en el caso de las algas, la autorregulación significa el aporte de nutrientes que reciben las algas y que regula su densidad. b) Representación gráfica de las predicciones del modelo como respuesta a un impacto positivo que aumente la abundancia/biomasa de peces. Este impacto provocará la disminución de la población de erizos (círculo pequeño) y, en consecuencia, el incremento de las algas (círculo grande)

Fig. 1. a) Model signed-digraph from a simple trophic system of three variables ( $\mathrm{P}=$ Fish, $\mathrm{E}=\mathrm{Sea}$ urchin, and $\mathrm{A}=\mathrm{Alga}$ ). Negative self-regulation for fish means that the population consumes alternative prey not included in the model; in the case of alga, self-regulation refers to the nutrient contribution received by alga, regulating density. b) Graphic representation of the model's predictions as a response to a positive impact that would increase fish abundance/biomass. This impact decreases the sea urchin population (small circle) and consequently increases alga (large circle)

$$
\begin{aligned}
& \text { (j) } \\
& \text { P E A } \\
& \begin{array}{cccccccc} 
& \mathrm{P} & \mathrm{a}_{\mathrm{PP}} & \mathrm{a}_{\mathrm{PE}} & \mathrm{a}_{\mathrm{PA}} & -1 & 1 & 0 \\
\mathrm{~A}=\text { (i) } & \mathrm{E} & \mathrm{a}_{\mathrm{EP}} & \mathrm{a}_{\mathrm{EE}} & \mathrm{a}_{\mathrm{EA}}= & -1 & 0 & 1 \\
& \mathrm{~A} & \mathrm{a}_{\mathrm{AP}} & \mathrm{a}_{\mathrm{AE}} & \mathrm{a}_{\mathrm{AA}} & 0 & -1 & -1
\end{array}
\end{aligned}
$$

Una vez generada la matriz de la comunidad, se puede evaluar el comportamiento del sistema ante un impacto o disturbio (positivo o negativo). En esta técnica se define como impacto aquel factor externo que causa un incremento (disturbio positivo) o decremento (disturbio negativo) en la variable afectada. La respuesta de cada variable del sistema ante el impacto se resume en una matriz de predicciones que se calcula como la matriz adjunta de la matriz de la comunidad (adj-A) (Puccia \& Levins, 1985). 
En el ejemplo peces-erizos-algas, la matriz de predicción ante un disturbio positivo que afecta cada variable del sistema sería:

$$
\begin{array}{rrrrr} 
& \mathrm{P} & \mathrm{E} & \mathrm{A} \\
\mathrm{P} & 1 & 1 & 1 \\
\operatorname{adj-\mathbf {A}}= & \mathrm{E} & -1 & 1 & 1
\end{array}
$$

\section{A $\quad \begin{array}{lll}1 & -1 & 1\end{array}$}

La respuesta de las variables en la matriz de predicción se lee por columnas y el signo de los valores son interpretados como un aumento $(+)$, disminución (-) o no cambio (0) en la biomasa o abundancia de las poblaciones. Así, la presencia de un factor externo que aumente la densidad/biomasa de peces (1) (primera columna de la matriz de predicción del ejemplo) provocará la disminución de la población de erizos (-1) y, en consecuencia, el incremento de las algas (1) (Fig. 1b). A pesar de que la variable algas (A) no está directamente conectada con la variable que recibe el impacto $(\mathrm{P})$, el efecto del impacto se transmite de manera indirecta a través de los erizos. Para asignar un valor de probabilidad a las predicciones asignadas, Dambacher et al. (2002) desarrollaron la matriz de predicción "ponderada" (weighted prediction en inglés), la cual permite elegir entre aquellos modelos con predicciones significativas $(\geq 0.4)$ $\mathrm{y}$ aquellos con predicciones débiles o poco significativas $(<0.4)$. Para el ejercicio de la figura 1 la matriz de predicción ponderada [W] indica que todas las predicciones tienen una alta probabilidad de ocurrir:

$$
\text { P E A }
$$

$$
\begin{array}{llll}
\mathrm{P} & 1 & 1 & 1 \\
\mathrm{~W}=\mathrm{E} & 1 & 1 & 1
\end{array}
$$

$$
\text { A } \quad 1 \quad 1 \quad 1
$$

En Loop Analysis se define como estabilidad la capacidad de un sistema de regresar a los niveles de equilibrio que prevalecían antes de un disturbio. Si los niveles de equilibrio aumentan o disminuyen sin límites, oscilando permanentemente, el sistema es considerado inestable (Dambacher et al. 2003). Para determinar la estabilidad de los sistemas propuestos se utilizaron las pruebas de coeficientes (F0) y el criterio de Hurwitz, sugeridos por Puccia \& Levins (1985). La descripción detallada del funcionamiento de Loop Analysis y su fortaleza matemática se puede consultar en Puccia \& Levins (1985) y Dambacher et al. (2002).

\section{Construcción del modelo}

Las variables del modelo se seleccionaron por su importancia como recurso pesquero y su abundancia en la captura incidental (Arreguín-Sánchez et al. 2002). Cada grupo o variable pesquera está formada por las especies que se capturan con el mismo arte de pesca; por ejemplo, los moluscos se capturan con buceo. Se utilizaron criterios pesqueros y de conservación (e.g., vedas o declaración de especies 
con algún estatus de protección) para agrupar en siete variables y tres categorías a las especies relacionadas directa o indirectamente con la pesca en el NGC: a) Especies objetivo capturadas por lo menos con un arte de pesca $(\mathrm{Cr}=$ crustáceos, $\mathrm{M}=$ moluscos, Es=escama, El=elasmobranquios), b) Captura incidental de especies que no son el recurso objetivo de una pesquería pero que no siempre se descartan porque llegan a tener valor comercial $(\mathrm{CIO}=$ omnívora $\quad$ y $\mathrm{CIC}=$ carnívora $)$, y c) Especies protegidas (SPr) por algún estatus legal de protección nacional o internacional debido a su estado poblacional crítico (riesgos de extinción, endémicos o amenazados) y que están indirectamente relacionadas con la pesca por su captura incidental (Cuadro 1). Además, para poder discutir las predicciones de los modelos desde una perspectiva de la planeación ambiental (Espinoza-Tenorio et al. 2010) y que los resultados pudieran ser utilizados para identificar nuevas líneas de trabajo académico, hipótesis de investigación o vislumbrar opciones en las estrategias del MBE (Bondavalli et al. 2009; Gregory et al. 2013), se utilizó también la definición de indicadores ambientales propuesta por la Organización para la Cooperación y el Desarrollo Económico (OECD, 1993). De este modo, se seleccionaron como variables del modelo únicamente aquellas que cumplieran los requisitos de validez analítica, facilidad de medición y relevancia política.

Para seleccionar las relaciones entre variables se utilizaron como criterios los hábitos alimenticios y el nivel trófico de las especies más abundantes de acuerdo con el conocimiento ecológico local (Cuadro 2). Los programas usados en esta sección fueron: PowerPlay Digraph Editor versión 2.0 y Maple versión 5.00 .

El modelo de la comunidad biológica del NGC (Fig. 2) representa un sistema que, en términos teóricos, no ha sido afectado por la pesca o un escenario de protección extrema, y donde la pesca es un disturbio puntual, controlado y externo. Esto permitió analizar las predicciones del modelo ante la disminución de la abundancia o biomasa de los recursos debido a un impacto en cada una de las variables (e.g., incremento en la pesca de moluscos, captura incidental).

\section{RESULTADOS}

Construcción del modelo de la comunidad biológica del NGC

El modelo de la comunidad biológica del NGC se construyó con un total de 83 especies relacionadas directa o indirectamente con la pesca (Cuadro 1). De los recursos pesqueros se encontró que la producción (entre 1993-1998) de crustáceos, que se basa primordialmente en dos especies, fue la más importante $(23000 \mathrm{t})$, seguida por la pesca de especies de escama (21 700 t). En cambio, la pesca de moluscos fue la que menos volúmenes de extracción aportó, pero fue la más diversa (10 especies). Entre las especies relacionadas con la pesca 
Cuadro 2. Información trófica sobre la comunidad biológica del Norte del Golfo de California, México

Table 2. Trophic information on the biological community of the Northern Gulf of California, Mexico

\begin{tabular}{|c|c|c|c|}
\hline & & $\begin{array}{l}\text { Principales } \\
\text { alimenticios }\end{array}$ & $\begin{array}{l}\text { Fuentes } \\
\text { información }\end{array}$ \\
\hline \multirow{2}{*}{$\begin{array}{l}\text { a) Depredadores } \\
\text { tope }\end{array}$} & Especies protegidas & $\begin{array}{l}\text { Carnívoros } \\
\text { (peces, moluscos, } \\
\text { crustáceos) v omnívoros }\end{array}$ & $\begin{array}{l}1,3,5,9,10,11,13 / \\
\text { I, II, IV }\end{array}$ \\
\hline & Elasmobranquios & $\begin{array}{l}\text { Carnívoros (moluscos y } \\
\text { peces) }\end{array}$ & $\begin{array}{l}4,8,10,11,14 \text { / I, IV, V, } \\
\text { VI, VII }\end{array}$ \\
\hline \multirow{2}{*}{$\begin{array}{l}\text { b) Carnívoros } \\
\text { secundarios }\end{array}$} & Escama & Carnívoros (peces) & $2,10,11,14$ / I, II, IV \\
\hline & $\begin{array}{l}\text { Captura incidental } \\
\text { carnívora }\end{array}$ & Peces y crustáceos & $2,10,11,14$ / I, III, IV \\
\hline \multirow{3}{*}{ c) Base trófica } & Crustáceos & $\begin{array}{l}\text { Filtradores y carnívoros } \\
\text { (otros crustáceos) }\end{array}$ & 10, 11 / I, IV, VIII \\
\hline & Moluscos & Filtradores y carnívoros & $\begin{array}{l}6,7,10,11,12 \text { / I, III, } \\
\text { IV }\end{array}$ \\
\hline & $\begin{array}{l}\text { Captura incidental } \\
\text { omnívora }\end{array}$ & $\begin{array}{l}\text { Peces, poliquetos, algas, } \\
\text { detritus y microalgas }\end{array}$ & $2,10,11,14$ / I, III, IV \\
\hline
\end{tabular}

(Fuentes de información. Cuantitativa: a) Literatura publicada: 1. Aurioles-Gamboa \& Zavala-González (1994); 2. Allen et al. (1995); 3. Jefferson \& Leatherwood (1995); 4. Compagno et al. (1995); 5. Márquez-Márquez (1995); 6-7. Poutiers (1995a; 1995b); 8. MárquezFarías (2000); 9. Pedrín-Osuna et al. (2001); 10. Arreguín-Sánchez et al. (2002); 11. Morales-Zárate et al. (2004), b) Literatura gris: 12. Carmona (1993); 13. García-Rodríguez (1999); 14. Froese \& Pauly (2011). Cualitativa: a) Informantes clave: I. Pedrín, O. antes con INP (comunicación personal, enero, 2004); II. Cisneros-Mata, M. A. WWF (comunicación personal, enero, 2004); III. García, A. R. CRIPGuaymas (comunicación personal, enero, 2004); IV. Lercari, D. Posgrado-CICIMAR (comunicación personal, febrero, 2004); V. SosaNishizaki, O. y VI. Pérez, J. C. CICESE (comunicación personal, febrero, 2004), b) Grupos especializados: VII. Grupo de pesquerías de tiburón (CICESE); VIII. Investigadores, CRIP-Guaymas).

predominan los hábitos alimenticios carnívoros (al menos en un estadio de su vida) y estas se distribuyen en al menos tres niveles tróficos: base trófica, carnívoros secundarios y depredadores tope (Cuadro 2).

La captura incidental fue el grupo más numeroso, ya que incluyó 51 especies, de las cuales la mayoría (40) son peces carnívoros secundarios. Este grupo fue el que presentó más relaciones depredador-presa (tres como presa y dos como depredador). Aunque los depredadores tope tienen hábitos principalmente ictiófagos, la diversidad del grupo de especies protegidas (mamíferos, peces $\mathrm{y}$ tortugas) resultó en un amplio espectro de hábitos alimenticios.

\section{Respuesta de la comunidad biológica ante la explotación pesquera}

En general, las predicciones sugieren que la explotación de los grupos que se ubican en la parte baja de la trama trófica, crustáceos $(\mathrm{Cr})$, moluscos (M) y captura incidental omnívora (CIO), causaría una disminución en cuatro grupos pesqueros (Fig. 3a, b y c); por el contrario, la explotación de la escama (Es), captura incidental de carnívoros (CIC) y elasmobranquios (El) causaría la disminución de tres grupos (Fig. 

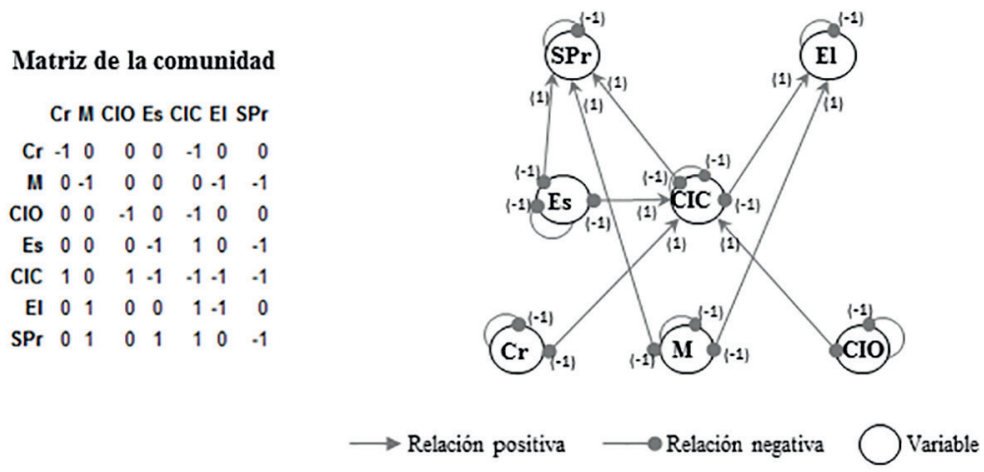

Fig. 2. Modelo de la comunidad biológica del Norte del Golfo de California, México, construido a partir de las principales relaciones entre las variables de los recursos pesqueros (Cuadro 2). $\mathrm{Cr}=$ Crustáceos; $\mathrm{M}=$ Moluscos; Es=Escama; $\mathrm{El}=$ Elasmobranquios; $\mathrm{CIO}=\mathrm{Captura}$ incidental omnívora; $\mathrm{CIC}=$ Captura incidental carnívora; $\mathrm{SPr}=$ Especies protegidas

Fig. 2. Model signed-digraph for biological community of the Northern Gulf of California, Mexico, made from the main interactions between variables of fisheries resources (Table 2). Cr=Crustaceans; M=Mollusks; Es=Fish; El=Elasmobranchs; $\mathrm{CIO}=$ Omnivore By-Catch; $\mathrm{CIC}=$ Carnivore By-Catch; SPr=Protected Species

3d, e y f). Es interesante notar que los grupos pesqueros que aumentan o disminuyen en diferentes escenarios de explotación son diferentes (Fig. 3); por ejemplo, la pesca de crustáceos promueve que aumenten las poblaciones de moluscos (M) y de la captura incidental omnívora (CIO) (Fig. 3a); por el contrario, si la pesca se realiza sobre moluscos, las poblaciones que aumentan son el grupo escama (Es) y la captura incidental carnívora (CIC) (Fig. 3b).

El análisis de las predicciones permitió conocer los efectos tanto directos como indirectos de la remoción por pesca de los diferentes grupos incluidos en el modelo. Por ejemplo, dentro de los carnívoros secundarios (Es y CIC) se encontraron dos efectos. Por un lado, las especies de captura incidental carnívora (CIC) tienen una posición clave en el flujo de la trama trófica, ya que al disminuir estas (Fig. 3e), directamente se reducen otras poblaciones carnívoras (El y SPr) que dependen de las especies de este grupo como alimento (efecto indirecto). Esta disminución de depredadores tope promueve indirectamente el aumento de sus presas $(\mathrm{M})$.

Otro ejemplo de los efectos directos e indirectos se observa en la disminución del recurso escama (Es) (Fig. 3d), cuya explotación ocasionaría un aumento directo en CIC (su único alimento) y una disminución indirecta en grupos de niveles tróficos más bajos ( $\mathrm{Cr}$ y $\mathrm{CIO}$ ), los cuales son presa de CIC, así como la disminución de las especies protegidas, que dependen del recurso escama que disminuyen por la explotación.

Los moluscos son elúnico grupo que ante cualquier escenario de explotación 


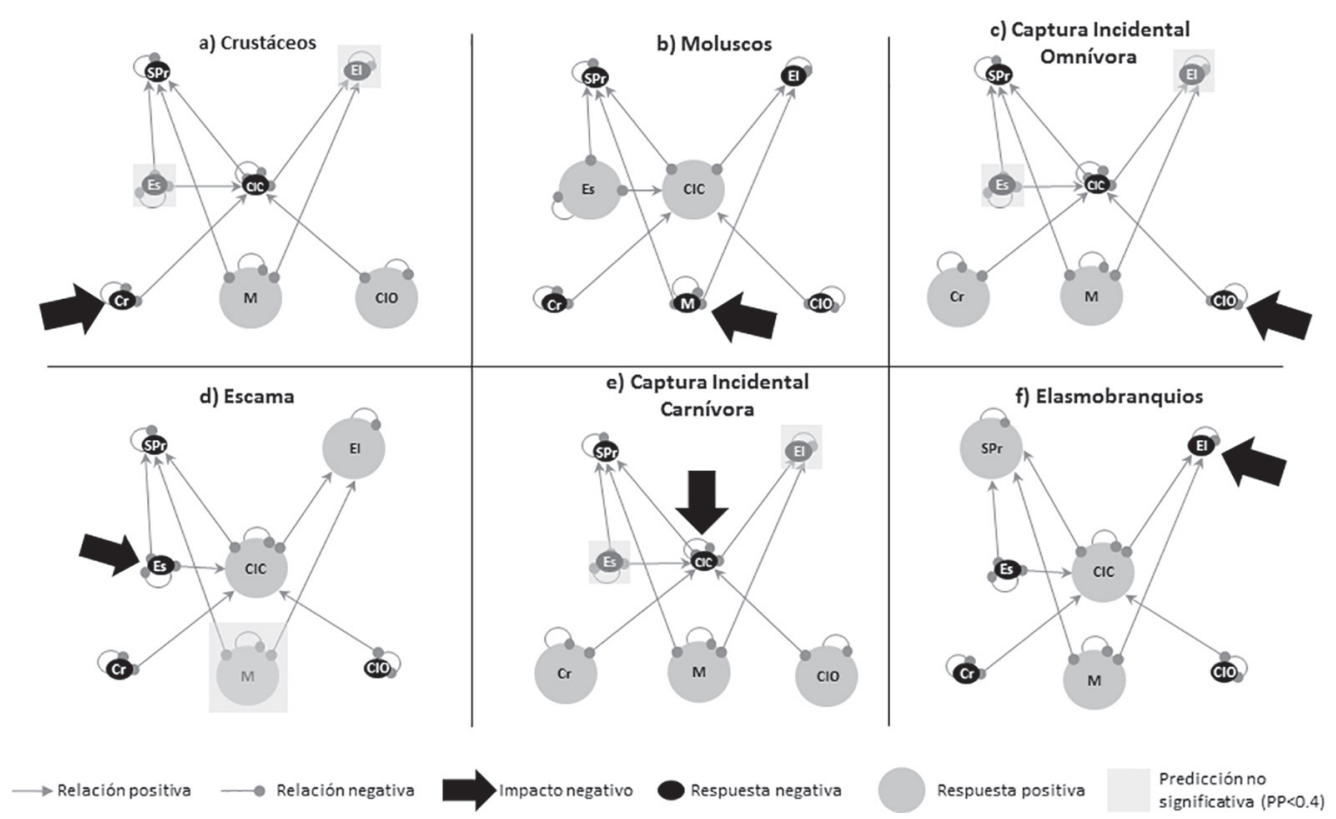

Fig. 3. Predicciones del modelo de la comunidad biológica del Norte del Golfo de California, México (Fig. 2). Cada diagrama, incisos a) a f), representa las predicciones del modelo como respuesta a la disminución de cada variable debida a la explotación pesquera (flecha negra). El tamaño de los círculos indica la dirección de la respuesta: círculos grandes=aumenta la densidad/biomasa; círculos pequeños=disminuye la densidad/biomasa

Fig. 3. Predictions from the model signed-digraph for the biological community of the Northern Gulf of California, Mexico (Fig. 2). Each diagram represents the model predictions as a response to the decrease of each variable due to fishery (black arrow). The size of the circles indicates the direction of the response: large circles=density/ biomass increase; small circles $=$ density $/$ biomass decrease

de los diferentes grupos pesqueros, estos (moluscos) tenderían a aumentar. En contraste, los elasmobranquios (El) y las especies protegidas (SPr) disminuyen en todos los escenarios pesqueros, a excepción de la pesca de escama (Es), en donde aumenta El y la pesca de elasmobranquios, en la que aumenta SPr (Fig. 3).

Los efectos indirectos de la explotación aparecen en todos los escenarios pesqueros, observándose en todos los casos el efecto en diferentes niveles tróficos (Fig. 3). De particular interés es la respuesta del sistema ante la explotación de recursos de niveles tróficos bajos (Cr, M y CIO), ya que en todos los casos las especies protegidas (SPr) y los elasmobranquios (El) disminuyen (Fig. 3a, b y c). El patrón de respuestas no es tan claro ante la explotación de recursos de niveles tróficos intermedios y altos (Fig. 3d, e y f).

\section{DISCUSIÓN}

Loop Analysis ha mostrado su versatilidad para la construcción de modelos tróficos capaces de sugerir 
respuestas -obvias y no tan obviasde las variables de un sistema ante un impacto. En cerca de dos décadas de trabajo una diversidad de sistemas acuáticos han sido modelados (e.g., lagunas y esteros costeros, comunidades marinas bénticas), con los cuales se ha buscado comprender cómo la estructura (número de variables, interconexiones entre ellas y mecanismos de retroalimentación) de las comunidades biológicas influye en su respuesta a algunos tipos de impactos ambientales (Loiselle et al. 2000; Montaño-Moctezuma et al. 2007), y qué implicaciones tienen estas respuestas para la conservación y uso de la biodiversidad (Bodini et al. 1994; Ortiz \& Wolff, 2002; Ortiz, 2003; Ortiz et al. 2013). Otras aproximaciones han representado la dinámica espacial y temporal de los ecosistemas sujetos a pesca (Espinoza-Tenorio et al. 2010; 2013), considerando diferentes niveles de explotación y conservación (Montaño-Moctezuma et al. 2008).

En este estudio se enfatiza la factibilidad de utilizar el conocimiento ecológico local (CEL), como información complementaria que permitió elaborar un modelo para representar la comunidad biológica del NGC. Las predicciones del modelo propuesto permitieron conocer los efectos directos e indirectos de la explotación de diferentes grupos pesqueros, identificando las pesquerías que pueden presentar conflicto debido a que algunas explotan recursos que son depredadores en el sistema y otras pesquerías que extraen a sus presas. El conocer cómo responde cada grupo pesquero ante la remoción de los diferentes recursos facilitará a los distintos usuarios del recurso, así como a las instituciones encargadas de la conservación, discernir entre estrategias de manejo que consideren tanto los objetivos de utilización de los recursos como aquellas orientadas a la conservación, observando en ambos casos, los posibles efectos directos e indirectos de la pesca en el ecosistema.

\section{Representatividad del modelo}

El modelo se construyó con una base de fuentes similar a la utilizada por los modelos cuantitativos para la misma área (Arreguín-Sánchez et al. 2002; Morales-Zárate et al. 2004; Lercari, 2006), pero integró también la parte cualitativa del conocimiento ecológico local (CEL), la cual permitió complementar y validar los datos científicos con base en la experiencia de los usuarios locales. Aunque el CEL es una herramienta que ha probado su utilidad en otros casos de MBE (Olsson \& Folke, 2001; Berkes \& Folke, 2002), en el NGC se debe considerar que el conocimiento empírico no se ha aplicado de manera integrada, ya que la prioridad ha sido el conocimiento sobre las especies protegidas, las cuales motivaron la existencia del ANP; lo mismo sucede con ciertas pesquerías de importancia comercial que son más conocidas.

La versatilidad de Loop Analysis permite analizar un sistema parcial o subsistema que no incluye a todos los niveles de la trama alimenticia, ya que 
asume que el subsistema descrito es parte de un sistema estable que está conectado al modelo principal en forma de retroalimentaciones negativas que comienzan y terminan en las variables consideradas, indicando una relación de denso-dependencia (Puccia \& Levins, 1985). Un ejemplo sería la conexión del grupo de los moluscos, crustáceos y captura incidental omnívora con sus fuentes de alimento (fitoplancton, zooplancton, otros moluscos y crustáceos, etc.), que no fueron incluidas en el modelo en forma de variables (Fig. 2). Acercamientos que permiten el estudio de dichas variaciones biológicas y pesqueras en el NGC se pueden encontrar en EspinozaTenorio et al. (2010) y Lercari (2006).

Aunque nuestro modelo fue una representación simplificada de la estructura trófica de la comunidad relacionada con la pesca en el NGC, los siete grupos funcionales del modelo propuesto contienen gran parte de los 29 utilizados por Morales-Zárate et al. (2004) para representar el ecosistema entero, o los 27 empleados por Arreguín-Sánchez et al. (2002) para el ecosistema béntico de todo el Golfo de California. Los grupos funcionales que no concuerdan con estos dos modelos cuantitativos se debe a que no están relacionados directamente con la pesca regional, ya que pertenecen en su mayoría a la base de la trama trófica (detritus, fitoplancton, zooplancton, poliquetos, estomapatópodos y macrofitas), no son especies acuáticas (aves marinas) o son organismos cuya principal zona de distribución no es el
NGC (ballenas dentadas y barbadas y peces de las familias Clupeidoae, Triglidae y Myctophidae).

La estructura que se usó para representar la comunidad biológica del NGC es también similar a las documentadas por otras investigaciones del mismo ecosistema. Por ejemplo, el grupo de captura incidental incluyó 51 especies que son reportadas oficialmente, pero su número es similar al encontrado en otros estudios que han identificado hasta 67 especies (Pérez-Mellado \& Findley, 1985) y que, a diferencia de nuestro trabajo, incluyen especies que en general no se registran en las estadísticas pesqueras. Además, se encontró que la mayoría de las especies de este grupo son carnívoras y peces, lo cual coincide con lo reportado por Hendrickx (1985), quien menciona que la mayoría de las especies de la captura incidental para el Golfo de California la integran peces y otros grupos (camarones mantis, esquilas o catalinas de mar). De manera general, también el número de especies objetivo (Cuadro 1a; Fig. 1) que representa cada nivel trófico, en nuestro modelo coincide con la estructura pesquera piramidal propuesta por Pauly \& Christensen (1995) para sistemas costeros. Según estos autores, es en la base, donde son más abundantes las especies aprovechadas por las pesquerías, lo cual coincide con el mayor número de especies objetivo que fueron agrupadas en los niveles tróficos inferiores $(\mathrm{Cr}=5$ y $\mathrm{M}=10)$, en las especies carnívoras secundarias 
$(E s=5)$ o depredadores tope $(E l=5)$. En términos de producción, también los crustáceos en la parte baja de la estructura trófica mostraron esta tendencia de mayor explotación, aunque el aporte a la pesquería del grupo escama fue significativo.

\section{Implicaciones ecosistémicas para el manejo del NGC}

Los resultados de este estudio permiten identificar las posibles consecuencias directas e indirectas de la explotación de diferentes grupos pesqueros en el ecosistema del NGC. Esta visión estructurada del conocimiento empírico de la comunidad biológica-pesquera del NGC permite plantear las siguientes ideas:

a) La pesca de moluscos es considerada actualmente como la de mayor potencial de crecimiento para la zona (DOF, 2004). Las predicciones del modelo propuesto sugieren que en cualquier escenario pesquero, el grupo de los moluscos aumentaría, de tal manera que su explotación podría ser una estrategia loable. Sin embargo, al disminuir las poblaciones de moluscos también disminuirían directa e indirectamente varios grupos que forman parte del sistema. Algunos de estos grupos, como los crustáceos y elasmobranquios son las especies objetivo más importantes de la captura comercial en la región (Cuadro 1). Si esta estrategia orientada al aprovechamiento intensivo de los moluscos es promovida, sería esencial considerar la suma de efectos causados directamente por la pesquería sobre crustáceos (Cr) y elasmobranquios (El), más los efectos indirectos debidos a la explotación de moluscos.

b) Los carnívoros secundarios están en una posición clave de la trama trófica en todo el Golfo de California (Arreguín-Sánchez et al. 2002; Espinoza-Tenorio et al. 2010); el modelo regional aquí presentado muestra que las especies carnívoras de la captura incidental (CIC), son las que tienen más interacciones con el resto de las variables. La magnificación de un impacto puntual a través de interacciones no lineales o indirectas del sistema (Levin \& Lubchenco, 2008) sugiere que para el NGC la captura incidental es algo más que un retiro de recursos desaprovechados; por el contrario, su remoción constante representa un impacto que altera procesos tróficos, por lo que deteriora el estado de salud del ecosistema, especialmente de las especies protegidas que dependen de este grupo (CIC) como parte de su dieta. Sería recomendable conocer cuáles son las especies que representan la mayor proporción de la captura incidental y realizar un seguimiento de sus cambios en el tiempo, de tal manera que estas especies se consideren como indicadores para evaluar el estado del ecosistema.

c) Al igual que en el resto del Golfo de California (Sala et al. 2004), los resultados del modelo confirman que en el Norte del Golfo los niveles tróficos superiores (El y SPr) están siendo indirectamente afectados por la pesca. La explotación de crustáceos (23000 t) y la de escama (21 $700 \mathrm{t}$ ) reportada por SAGARPA en el periodo 1993-1998 
(Cuadro 1) fueron las más importantes en la región. Las predicciones de los modelos sugieren que la disminución de ambos grupos ( $\mathrm{Cr}$ y Es) tiene un efecto indirecto negativo sobre las especies protegidas. Los niveles tróficos superiores son en especial vulnerables porque sus poblaciones son menos elásticas (capacidad de recibir un disturbio y regresar a su estado original), ya que típicamente son especies grandes, de ciclo de vida largo y de baja fecundidad (Shepered \& Ransom, 2005). La mayoría de los escenarios pesqueros analizados sugieren que tanto las especies con estatus de protección como el grupo de los elasmobranquios pueden disminuir. Se ha documentado (Bobadilla et al. 2011) que algunas de las especies protegidas consideradas en este grupo (e.g., vaquita marina) no muestran signos de recuperación; es probable que en estos casos los efectos indirectos causados por la pesca no permitan que estas especies se recuperen, a pesar de los esfuerzos de protección que se han implementado en la región.

d) El conocer los grupos pesqueros que potencialmente presentan un conflicto al ser explotados de forma simultánea es de utilidad para los usuarios de los diferentes recursos en la zona (pescadores e interesados en la conservación). Si las propuestas se presentan a los actores interesados en el área (gobierno, pescadores, $\mathrm{ONG}^{\prime}$ s, etc.), de tal manera que entre todos seleccionen una o varias estrategias de manejo, estas podrían ser aplicadas de forma experimental en una zona particular. De modo complementario, sería importante implementar un programa de monitoreo que permita validar y ajustar el modelo propuesto, es decir, conocer si las predicciones del modelo se cumplen.

e) Si bien en la realidad es posible que la explotación de los diferentes grupos pesqueros ocurra en forma simultánea, resulta importante resaltar el efecto independiente de cada grupo, es por ello que el propósito de este ejercicio fue conocer el efecto particular de la explotación en los diferentes grupos pesqueros. El uso de esta metodología pretende facilitar la visualización de la complejidad de los ecosistemas que a su vez simplificaría la decisión sobre varias alternativas de explotación. Es decir, los modelos como herramienta de apoyo a los usuarios para discernir entre mejores estrategias de manejo que tomen en cuenta visiones económicas o de conservación.

\section{CONCLUSIONES}

Es evidente que existe un conflicto entre la explotación de los recursos de diferentes niveles tróficos debido a la interconexión de los sistemas ecológicos. Los objetivos del MBE no están orientados a impedir la explotación, por el contrario, los ejercicios académicos realizados en diferentes ecosistemas presentan diversas opciones para identificar a los grupos explotados y no explotados que podrían estar relacionados de manera directa o indirecta con el ecosistema. Esta información es importante para establecer objetivos tanto de utilización de los recursos como de conservación, y permite tener una guía sobre la cual 
elaborar propuestas de manejo basadas en el conocimiento del ecosistema.

El diseñar los modelos con la retroalimentación de los usuarios sirve además para motivar la educación ambiental sobre el tema de cadenas tróficas y de ecología marina y pesquera; del mismo modo, ayuda a explicar a los usuarios de los recursos sobre el efecto de la pesca en el ecosistema. Este mayor involucramiento de la sociedad en la construcción de las herramientas del MBE facilita los procesos de gobernanza, uno de los principales retos en el MBE (Ruckelshaus et al. 2008).

\section{AGRADECIMIENTOS}

Este estudio fue financiado por CONACYT por la beca de maestría a AET y por el proyecto SEMARNAT2002-C01-1313. Agradecemos también a las instituciones que nos apoyaron con su información y experiencia: la Reserva de la Biosfera AGCyRC, Grupo de Pesquerías CICESE, CRIPGuaymas, CEDO Intercultural, CoBi, Conservación Internacional, WWF y Pronatura-Sonora.

\section{BIBLIOGRAFÍA}

Allen, G. R., Bauchot, M. L., Bellwood, D. R., Bianchi, G., Bussing, W. A., Caruso, J. H., ... Whitehead, P. J. P. (1995). Peces óseos. En W. Fisher, F. Krup, W. Schneider, C. Sommer, K. E. Carpenter \& V. H. Niem (Eds.), Guía FAO para la identificación de especies para los fines de la pesca. Pacífico centro-oriental (pp. 799-1195). Roma, Italia: Food and Agriculture Organization of the United Nations.
Arreguín-Sánchez, F., Arcos, E. \& Chávez, E. A. (2002). Flows of biomass and structure in an exploited benthic ecosystem in the Gulf of California, Mexico. Ecol. Model., 156, 167-183. doi: 10.1016/S0304-3800(02)00159-X Aurioles-Gamboa, D. \& Zavala-González, A. (1994). Some ecological factors that establish distribution and abundance of the sea wolf Zalophus californianus in the California Gulf. Cienc. Mar., 20, 535-553.

Berkes, F. \& Folke, C. (2002). Back to the future: Ecosystem dynamics and local knowledge. En L. H. Gundrson \& C. S. Holling (Eds.), Panarchy, understanding transformations in human and natural systems (pp. 121-144). Washington-Covelo-London, EE. UU.: Island Press.

Bobadilla, M. S., Álvarez-Borrego, S., Ávila-Foucat, S., Lara-Valencia, F. \& Espejel, I. (2011). Evolution of environmental policy instruments implemented for the protection of totoaba and the vaquita porpoise in the Upper Gulf of California. Envir. Sci. Policy, 14, 998-1007. doi: 10.1016/j. envsci.2011.06.003

Bodini, A., Giavelli, G. \& Rossi, O. (1994). The qualitative analysis of community food webs: Implications for wildlife management and conservation. J. Envir. Manage., 41, 49-65. doi: 10.1006/jema.1994.1033

Bondavalli, C., Favilla, S. \& Bodini, A. (2009). Quantitative versus qualitative modeling: A complementary approach in ecosystem study. Comput. Biol. Chem., 33(1), 22-28. doi: 10.1016/j. compbiolchem.2008.07.012

Caddy, J. F. \& Seijo, C. J. (2005). This is more difficult than we thought! The responsibility of scientists, managers and stakeholders to mitigate 
the unsustainability of marine fisheries. Philos. T. R. S., 360, 59-75. doi: 10.1098/rstb.2004.1567

Carmona, C. M. C. (1993). Estructura de la comunidad bentónica en la porción Norte del Golfo de California (Primavera, 1990). Tesis de Licenciatura no publicada, Facultad de Ciencias Marinas-UABC, Ensenada, México.

CBD. Convention on Biological Diversity. (2003). Review of the principles of the ecosystem approach and suggestions for refinement: A framework for discussion. Montreal, Canada: Convention on Biological Diversity.

Cinti, A., Duberstein, J. N., Torreblanca, E. \& Moreno-Báez, M. (2014). Overfishing drivers and opportunities for recovery in small-scale fisheries of the Midriff Islands region, Gulf of California, Mexico: The roles of land and sea institutions in fisheries sustainability. Ecol. Soc., 19(1), 15. doi: 10.5751/ ES-05570-190115

Cisneros-Mata, M. A., MontemayorLópez, G. \& Román-Rodríguez, M. J. (1995). Life history and conservation of Totoaba macdonaldi. Conserv. Biol., 9, 806-814. doi: 10.1046/j.1523-1739.1995.09040806.x CITES. (2011). The CITES species. Convention on International Trade in Endangered Species of Wild Fauna and Flora. Ginebra, Suiza. Recuperado el 21 de septiembre, 2011, disponible en www.cites.org/eng/disc/species.php

Compagno, L. J. V., Krupp, F. \& Schneider, W. (1995). Tiburones. En W. Fischer, F. Krup, W. Schneider, C. Sommer, K. E. Carpenter \& V. H. Niem (Eds.), Guía FAO para la identificación de especies para los fines de la pesca. Pacífico centro-oriental (pp. 647-744).
Roma, Italia: Food and Agriculture Organization of the United Nations.

Corkeron, J. P. (2006). Opposing views of the "ecosystem approach" to fisheries management. Conserv. Biol., 20, 617-619. doi: 10.1111/j.1523-1739.2006.00436.x

Cudney, B. R. \& Turk, B. P. J. (1998). Pescando entre mareas del Alto Golfo de California. Una guía sobre la pesca artesanal, su gente y sus propuestas de manejo. Pto. Peñasco, México: CIEDO.

Dambacher, J. M., Li, H. W. \& Rossignol, P. A. (2002). Relevance of community structure in assessing indeterminacy of ecological predictions. Ecology, 83, 1372-1385. doi: 10.1890/0012-9658(2002)083[1372:RO CSIA]2.0.CO;2

Dambacher, J. M., Li, H. W. \& Rossignol, P. A. (2003). Qualitative predictions in model ecosystems. Ecol. Model., 161, 79-93. doi: 10.1016/ S0304-3800(02)00295-8

DOF. Diario Oficial de la Federación. (1990). Se establece veda para las especies y subespecies de tortuga marina en aguas de jurisdicción federal del Golfo de México y Mar Caribe, asi como en las del Océano Pacífico, incluyendo el Golfo de California. Ciudad de México, México: Gobierno Federal Mexicano.

DOF. Diario Oficial de la Federación. (1994). Norma Oficial Mexicana-SEMARNAT-023-1993, por la que se establecen medidas para la protección de especies de totoaba y vaquita en agua de jurisdicción federal del Golfo de California. Ciudad de México, México: Gobierno Federal Mexicano.

DOF. Diario Oficial de la Federación. (2004). Mediante el cual se aprueba la actualización de la Carta Nacional Pesquera y su 
anexo. México. Ciudad de México, México: Gobierno Federal Mexicano.

DOF. Diario Oficial de la Federación. (2007). Ley General de Pesca y Acuacultura sustentables. Ciudad de México, México: Gobierno Federal Mexicano.

Espinoza-Tenorio, A., Montaño-Moctezuma, G. \& Espejel, I. (2010). Ecosystem-based analysis in a marine protected area where fisheries and protected species coexist. Environ. Manage., 45, 739-750. doi: 10.1007/ s00267-010-9451-0

Espinoza-Tenorio, A., Wolff, M., Taylor, M. H. \& Espejel, I. (2012). What model suits ecosystem-based fisheries management? A plea for a structured modeling process. Rev. Fish Biol. Fisher, 22, 81-94. doi: 10.1007/ s11160-011-9224-8

Espinoza-Tenorio, A., Wolff, M. \& Espejel, I. (2013). Using traditional ecological knowledge to improve holistic fisheries management: transdisciplinary modeling of a lagoon ecosystem of southern Mexico. Ecol. Soc., 18(2), 6. doi: 10.5751/ES-05369-180206

Finley, C. (2009). The social construction of fishing, 1949. Ecol. Soc., 14, 1-6.

Froese, R. \& Pauly, D. (2011). FishBase. World Wide Web electronic publication. Recuperado el 31 de agosto, 2011, disponible en www.fishbase.org

García-Rodríguez, F. J. (1999). Cambios espaciales y estacionales en la estructura trófica y consumo del lobo marino de California, Zalophus californianus, en la región de las grandes islas, Golfo de California. Tesis de Maestría no publicada, CICIMAR-IPN, La Paz, México.

Gilchrist, G., Mallory, M. \& Merkel, F. (2005). Can local ecological knowledge contribute to wildlife management?
Case studies of migratory birds. Ecol. Soc., 10(1), 20.

Gregory, A. J., Atkins, J. P., Burdon, D. \& Elliott, M. (2013). A problem structuring method for ecosystem-based management: The DPSIR modelling process. Eur. J. Oper. Res., 227(3), 558569. doi: 10.1016/j.ejor.2012.11.020

Hendrickx, M. E. (1985). Diversidad de los macroinvertebrados bentónicos acompañantes del camarón en el área del Golfo de California y su importancia como recurso potencial. En A. Yáñez-Arancibia (Ed.), Recursos pesqueros potenciales de México: la pesca acompañante del camarón (pp. 95-148). Mazatlán, México: Instituto de Ciencias del Mar y LimnologíaUNAM, Instituto Nacional de Pesca.

Hernández, A. \& Kempton, W. (2003). Changes in the fisheries management in Mexico: Effects of increasing scientific input and public participation. Ocean Coast. Manage., 46, 507-526. doi: 10.1016/S0964-5691(03)00032-2

IUCN. (2005). Islands and protected areas of the Gulf of California (Mexico). En IUCN World heritage nominationIUCN technical nomination (pp. 5567). Gland, Switzerland: International Union for Conservation of Nature and Natural Resources.

IUCN. (2011). The IUCN red list of threatened animals. Recuperado el 21 de septiembre, 2011, disponible en www.iucnredlist.org Jefferson, T. A. \& Leatherwood, S. (1995). Mamíferos marinos. En W. Fisher, F. Krup, W. Schneider, C. Sommer, K. E. Carpeneter \& V. H. Niem (Eds.), Guía FAO para la identificación de especies para los fines de la pesca. Pacifico centro-oriental (pp. 1669-1745). Roma, Italia: Food and Agriculture Organization of the United Nations. 
Lercari, B. D. (2006). Manejo de los recursos del ecosistema del Norte del Golfo de California: Integrando explotación y conservación. Tesis de doctorado no publicada, CICIMAR-IPN, La Paz, México.

Levin, S. A. \& Lubchenco, J. (2008). Resilience, robustness, and marine ecosystem-based management. BioScience, 58, 27-32. doi: 10.1641/B580107

Loiselle, S., Carpaneto, G. M., Hull, V., Waller, T. \& Rossi, C. (2000). Feedback analysis in reserve management: studying local myths using qualitative models. Ecol. Model., 129, 25-37. doi: 10.1016/S0304-3800(00)00217-9

Márquez-Márquez, R. (1995). Tortugas marinas. En W. Fisher, F. Krup, W. Schneider, C. Sommer, K. E. Carpeneter \& V. H. Niem (Eds.), Guía FAO para la identificación de especies para los fines de la pesca. Pacifico centro-oriental (pp. 16531664). Roma, Italia: Food and Agriculture Organization of the United Nations.

Márquez-Farías, J. F. (2000). Tiburones del Golfo de California. En M. A. M. Cisneros, M. F. L. Beléndez, B. E. Zárate, D. M. T. Gaspar, G. L. López del C., R. C. Saucedo \& A. J. Tovar (Eds.), Sustentabilidad y pesca responsable en México: Evaluación y manejo 19992000 (pp. 237-256). Ciudad de México, México: Instituto Nacional de Pesca.

Montaño-Moctezuma, G., Li, H. W. \& Rossignol, P. A. (2007). Alternative community interactions: a qualitative modeling approach. Ecol. Model., 205, 343-354. doi: 10.1016/j. ecolmodel.2007.02.031

Montaño-Moctezuma, G., Li., H. W. \& Rossignol, P. A. (2008). Variability of community interaction networks in kelp forest reserves and adjacent exploited areas. Fish. Res., 94(1), 99-108. doi: 10.1016/j.fishres.2008.07.003
Morales-Zárate, M. V., Arreguín-Sánchez, J., López-Martínez, S. E. \& LluchCota, E. (2004). Ecosystem trophic structure and energy flux in the Northern Gulf of California, Mexico. Ecol. Model., 174, 331-345. doi: 10.1016/j.ecolmodel.2003.09.028

OECD. (1993). OECD core set of indicators for environmental performance reviews. París, Francia: Organization for Economic Co-operation and Development.

Olsson, P. \& Folke, C. (2001). Local ecological knowledge and institutional dynamics for ecosystem management: a study of Lake Racken watershed, Sweden. Ecosystems, 4, 85-104. doi: 10.1007/s100210000061

Ortiz, M. \& Wolff, M. (2002). Application of loop analysis to benthic systems in northern Chile for the elaboration of sustainable management strategies. Mar. Ecol. Prog. Ser., 242, 15-27. doi: 10.3354/meps242015

Ortiz, M. (2003). Qualitative modeling of the kelp forest of Lessonia nigrescens Bory (Laminariales: Phaeophyta) in eulittoral marine ecosystems of the south-east Pacific: an approach to management plan assessment. Aquac., 220, 423-436. doi: 10.1016/ S0044-8486(02)00580-X

Ortiz, M., Levins, R., Campos, L., Berrios, F., Campos, F., Jordán, F. \& Rodríguez, F. (2013). Identifying keystone trophic groups in benthic ecosystems: Implications for fisheries management. Ecol. Ind., 25, 133140. doi: 10.1016/j.ecolind.2012.08.020

Ortiz-Lozano, L., Granados-Barba, A., Solís-Weiss, V. \& García-Salgado, M. A. (2005). Environmental evaluation and development problems of the Mexican Coastal Zone. Ocean Coast. Manage., 48, 161-176. doi: 10.1016/j. ocecoaman.2005.03.001 
Pauly, D. \& Christensen, V. (1995). Primary production required to sustain global fisheries. Nature, 374, 255-257. doi: $10.1038 / 374255 \mathrm{a} 0$

Pedrín-Osuna, O., Córdova-Murieta, H. J. \& Delgado-Marchena, M. (2001). Crecimiento y mortalidad de la totoaba, Totoaba macdonaldi, del alto golfo de California. Cienc. Pesquera, 14, 131-140.

Pérez-Mellado, J. L. \& Findley, L. T. (1985). Evaluación de la ictiofauna del camarón en las costas de Sonora y norte de Sinaloa, México. En A. YáñezArancibia (Ed.), Recursos pesqueros potenciales de México: La pesca acompañante del camarón (pp. 201-254). Ciudad de México, México: Universidad Nacional Autónoma de México.

Pikitch, E. K., Santora, C., Babcock, E. A., Bakun, A., Bonfil, R., Conover, D., ... \& Sainsbury, K. (2004). Ecosystem-based fishery management. Science, 305, 346347. doi: 10.1126/science.1098222

Plagányi, E. E. (2007). Models for an ecosystem approach to fisheries. Roma, Italia: Food and Agriculture Organization of the United Nations.

Poutiers, J. M. (1995a). Bivalvos. En W. Fisher, F. Krup, W. Schneider, C. Sommer, K. E. Carpeneter \& V. H. Niem (Eds.), Guía FAO para la identificación de especies para los fines de la pesca. Pacífico centro-oriental. Plantas e invertebrados (pp. 99-223). Roma, Italia: Food and Agriculture Organization of the United Nations.

Poutiers, J. M. (1995b). Gasterópodos. En W. Fisher, F. Krup, W. Schneider, C. Sommer, K. E. Carpeneter \& V. H. Niem (Eds.), Guía FAO para la identificación de especies para los fines de la pesca. Pacífico centro-oriental. Plantas e invertebrados (pp. 224-294). Roma, Italia: Food and Agriculture Organization of the United Nations.
Puccia, J. C. \& Levins, R. (1985). Qualitative modeling of complex systems: An introduction to loop analysis and time averaging. Cambridge, EE. UU.: Harvard University Press. doi: 10.4159/ harvard.9780674435070

Ruckelshaus, M., Klinger, T., Knowlton, N. \& DeMaster, D. P. (2008). Marine ecosystembased management in practice: scientific and governance challenges. BioScience, 58(1), 53. doi: 10.1641/B580110

SAGARPA. Secretaría de Agricultura, Ganadería, Desarrollo Rural, Pesca y Alimentación. (1998). Pesquerías comerciales (1993-1998) Oficina de Puerto Peñasco, Sonora; Golfo de Santa Clara y San Felipe, Baja California. Ciudad de México, México: Secretaría de Agricultura, Ganadería, Desarrollo Rural, Pesca y Alimentación.

Sala, E., Aburto-Oropeza, O., Reza, M., Paredes, G. \& López-Lemus, L. G. (2004). Fishing down coastal food webs in the Gulf of California. Fisheries, 29, 19-25. doi: 10.1577/1548-8446(2004)29[19:FD CFWI]2.0.CO;2

Shepered, D. T. \& Ransom, M. A. (2005). Direct and indirect fishery effects on small coastal elasmobranchs in the northern Gulf of Mexico. Ecol. Lett., 8, 1095-1104. doi: 10.1111/j.1461-0248.2005.00807.x

Taylor, S. J. \& Bogdan, R. (1990). Introducción a los métodos cualitativos de investigación, la búsqueda de significados. Buenos Aires, Argentina: Paidós.

Zárate, D. L., Saavedra, V. T., Rojas, G. J. L., Yáñez-Arancibia, A. \& Arriega, E. R. (1999). Terms of reference towards an integrated management policy in the coastal zone of the Gulf of Mexico and the Caribbean. Ocean Coast. Manage., 42, 345-368. doi: 10.1016/ S0964-5691(98)00060-X 